\title{
A RARE CASE OF CHRONIC ACRODERMATITIS ENTEROPATHICA PERSISTING AND RECURRING IN THE ADULT AGE
}

Rajkumar Kannan1, Vijayaanand Muthupandian², Balakrishnan Thenmozhi Priya ${ }^{3}$,Krishnaveni Alagar4, Deepika Loganathan 5

${ }^{1}$ Assistant Professor, Department of Dermatology, Kilpauk Medical College Hospital.
${ }^{2}$ Professor, Department of Dermatology, Kilpauk Medical College Hospital.
${ }^{3}$ Assistant Professor, Department of Dermatology, Kilpauk Medical College Hospital.
${ }^{4}$ Assistant Professor, Department of Dermatology, Kilpauk Medical College Hospital.
${ }^{5}$ Resident, Department of Dermatology, Kilpauk Medical College Hospital.

ABSTRACT

\section{BACKGROUND}

Acrodermatitis Enteropathica is a disorder of defective absorption of Zinc(1) that is inherited by an autosomal recessive mode of inheritance, characterised by a clinical triad of acral dermatitis, alopecia and diarrhoea. In majority of the patients, symptoms settle down at the time of puberty. Very rarely in certain cases, symptoms persist into the adult age, wherein it is underdiagnosed because of non-specific symptoms. We report a 26-year-old male with inherited zinc deficiency to highlight the persistence of isolated zinc deficiency into adult age, and also to stress the need of choosing the right chelating agent for the administration of oral elemental zinc, the combination of which crucially decides the better bioavailability of zinc, that could vary depending upon the chelating agent and genetically predetermined factors of the client.

\section{KEYWORDS}

Adult Acrodermatitis, Psoriasiform Lesions, Zinc Sulphate.

HOW TO CITE THIS ARTICLE: Kannan R, Muthupandian V, Priya BT, et al. A rare case of chronic acrodermatitis enteropathica persisting and recurring in the adult age. J. Evolution Med. Dent. Sci. 2016;5(98):7216-7218, DOI: 10.14260/jemds/2016/1632

\section{BACKGROUND}

Malabsorption of zinc can be inherited or acquired as encountered in certain nutritional disorders, chronic alcoholics, total parenteral nutrition, post-surgical status to bypass some of the upper intestine. Other causes include inflammatory bowel disease, pancreatic disease, necrolytic migratory erythema (Glucagonoma). Zinc belongs to the group of essential trace elements that includes iron, copper, manganese, nickel, cobalt, molybdenum, selenium, chromium, iodine, fluorine,(2) etc. Recommended dietary allowance for adults is $16 \mathrm{mg} /$ day. Zinc is indispensable to the normal functions of all cells, cellular systems, tissues and organs in the human body. It functions as the metal moiety of important enzymes viz, alkaline phosphatase, alcohol dehydrogenase and several different dehydrogenase and digestive enzymes.(3) Zinc also regulates DNA and RNA polymerases, thymidine kinase and ribonuclease and plays an important role in the immunological functions.

\section{CASE REPORT}

A 26-year-old male presented with Psoriasiform lesions over, peri-orificial, Perineal, Peri-anal areas and dorsum of hands \& feet of 3 months duration. History of similar lesions on and off since 7 months of age. Three months ago, patient developed a blister and an erosion spontaneously over the gluteal area. Later, patient developed similar erosions over the dorsum of

Financial or Other, Competing Interest: None.

Submission 04-11-2016, Peer Review 26-11-2016,

Acceptance 03-12-2016, Published 08-12-2016.

Corresponding Author:

Dr. Rajkumar Kannan,

Assistant Professor,

Department of Dermatology,

Kilpauk Medical College,

Kilpauk, Chennai-600010.

E-mail: rajderm0002@gmail.com

DOI: $10.14260 /$ jemds/2016/1632 hands and feet, Perineum and Periorificial areas extending into the malar areas of the cheeks. Patient gave a history of extremes of hair loss, fatiguability, fever with the eruption of pustules within the scaly patches. Angular cheilitis and soreness of mouth were present, along with delayed wound healing. No history of bowel disturbances. Patient had no comorbidities. On general examination, patient was looking thin built and had multiple well-defined psoriasiform scaly patches over perioral area; (Figure 1) a. Perineum and dorsum of hands. (Figure 1) b. Patches were studded with pustules. Diffuse hair loss over the scalp was there. Acral Psoriasiform lesions were studded with erosions and Crusting. Beau's lines were appreciated in the nails. Oral mucosa revealed extensive glossitis and candidosis. Haemoglobin was $15 \mathrm{~g} / \mathrm{dL}$. Other biochemical parameters viz., glycaemic profile, serology for human immunodeficiency virus were normal. Serum alkaline phosphatase was $58 \mathrm{u} / \mathrm{L}$. Serum zinc was $48.11 \mathrm{mg} / \mathrm{dL}$. (Normal range being $60-120 \mathrm{mg} / \mathrm{dL}$ ). Ultrasonogram of abdomen and upper GI endoscopy were normal; Duodenal mucosal biopsy revealed normal mucosal villi pattern, and PAS stain showed no additional findings. Serum Total protein and serum albumin were found to be normal.

Skin biopsy (Figure 2) revealed focal parakeratosis and superficial dermal infiltrate. Serum copper, B12 and iron levels were normal. Patient was treated with saline soaks to remove the crusting, and mid-potent steroid viz, mometasone furoate for the psoriasiform lesions. Antibiotic was Cephalexin $250 \mathrm{mg}$ thrice a day, and cap. zinc sulphate $140 \mathrm{mg}$ once a day for 10 days. Erosions dried well and there was no oozing of lesions within 4 days of commencement of treatment and healed completely within 10 days. It was practically found that patient responded faster and better to oral zinc sulphate than zinc acetate thereby highlighting the difference in the bioavailability of zinc varying with chelating agents used. 
Figure 1(a). Psoriasiform Lesion over Peri-oral Area

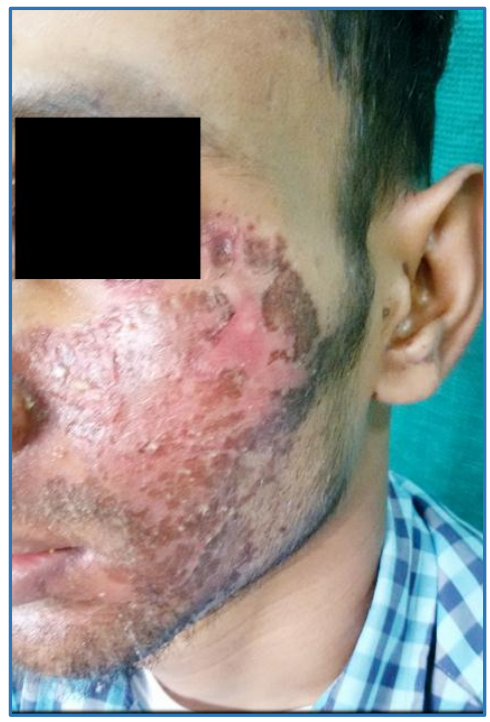

Figure 1(a). Before Treatment

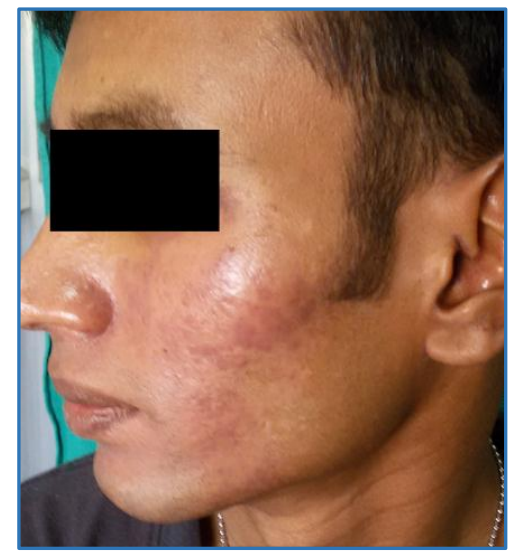

Figure 1(a). After Treatment

Figure 1(b). Psoriasiform Lesion over Hands

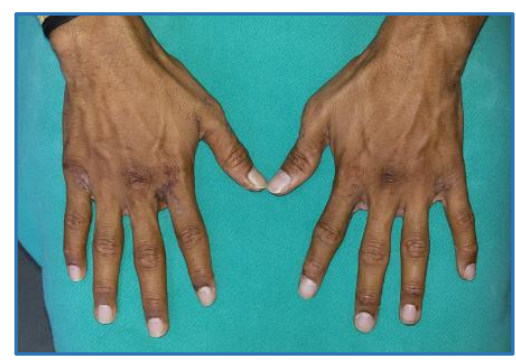

Figure 1(b). Before Treatment

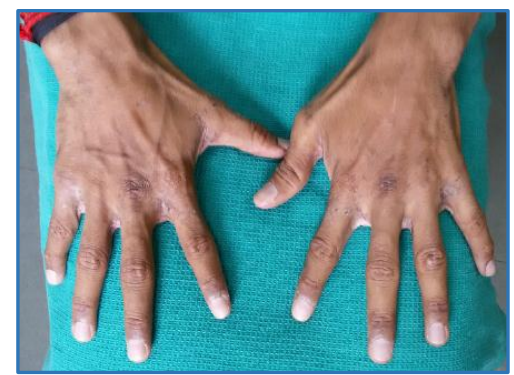

Figure 1(b). After Treatment

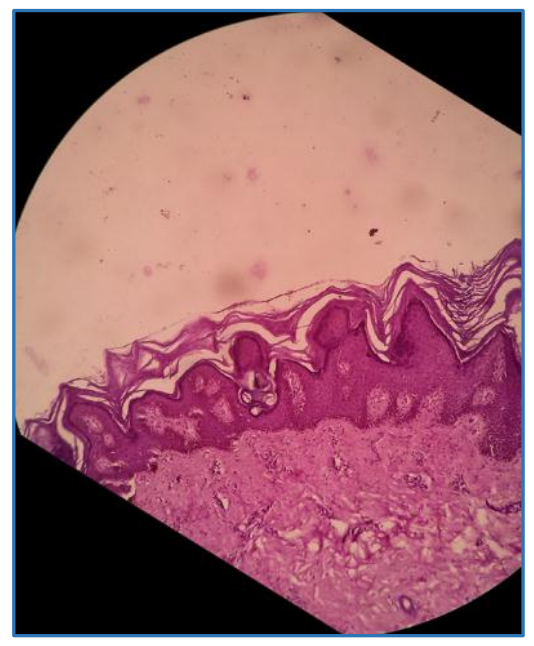

Figure 2. Skin Biopsy-H\&E Stain

- Focal Broad Parakeratosis.

- Superficial Dermal Inflammatory Infiltrate.

\section{DISCUSSION}

Acrodermatitis Enteropathica was first recognised in 1936 by Thore Brandt( $(4)$ and further investigated by Danbolt and Closs. (5) Congenital form of Acrodermatitis Enteropathica is a rare disorder beginning during infancy. The incidence is about 1 in 500,000 births and the condition affects males and females in equal numbers. Synonyms - Brandt Syndrome, DanboltCloss syndrome. The disease typically starts after weaning or earlier if the infant is not given breast milk. Child turns peevish, withdrawn and photophobic and develops a vesiculobullous dermatitis of hands, feet and periorificial areas. Scalp hair is lost. Wound healing is poor and skin lesions do not heal.(6)

Since its first discovery in an Iranian Male in 1961, Zinc deficiency in humans is now known to be an important malnutrition problems worldwide.(7)

Inherited form of zinc deficiency-due to malabsorption of zinc-genetic defect has been mapped to $8 \mathrm{q} 24$ and the defective gene identified as SLC39A4 which encodes the zinc transport protein, ZIP4.

A transient form-results due to failure of the mother to secrete zinc into her breast milk.

Acquired forms of zinc deficiency occur due to malabsorption syndromes like blind loop syndrome, inflammatory bowel disorders and post-intestinal resection status and total parenteral nutrition status without special zinc supplementation.

Symptoms of zinc deficiency other than dermatitis vary with age and can be diarrhoea, mood changes, anorexia and neurological disturbances. These were reported most frequently in infancy. Growth retardation, alopecia, weight loss and recurrent infections were prevalent in toddlers and school children, spontaneous remission may occur in adolescence.(8)

Zinc status in the human subjects is assessed by measurement of zinc in plasma, erythrocytes, neutrophils, lymphocytes and hair. (9)

Measurement of Plasma zinc is useful provided the plasma is unhaemolysed and conditions such as infections, acute 
stress and myocardial infarction and intravascular haemolysis are ruled out.

Available data indicate that zinc in neutrophils and the assay of activity of alkaline phosphatase in neutrophils may be the best tools for the diagnosis. Zinc supplements come in different chemical forms. Zinc alone is not easily absorbed from the intestines. To optimise the absorption it is chelated to amino acids like Zinc picolinate or made in inorganic form such as zinc sulphate or zinc oxide. It has been found that among the various chelating agents, zinc sulphate offers better elemental zinc absorption on oral administration even though on long term administration it may cause gastritis and related issues.

Our patient also responded faster and better to capsules of zinc sulphate than zinc acetate. Oral supplementation of zinc has been shown to be effective in the treatment of acrodermatitis enteropathica, say up to $220 \mathrm{mg}$ daily. Also it has been reported that high dosage of zinc treatment can lead to neurotoxicity, Alzheimer's disease or impaired lipid metabolism. ${ }^{(10)}$

As zinc plays a crucial role in immune regulation, chronic acrodermatitis enteropathica can lead on to secondary infections with pathogens like candida albicans, Staphylococcus aureus and Pseudomonas aeruginosa. Our patient had glossitis, angular cheilitis that are of candidal origin that responded well to treatment with oral fluconazole and topical clotrimazole oral paint.

This case is being reported to highlight the persistence of childhood acrodermatitis enteropathica into adult age and its tendency to relapse if not treated with life term supplementation of oral zinc.

\section{REFERENCES}

1. Weismann K, Hoe S, Knudsen L, et al. 65 Zinc absorption in patients suffering from acrodermatitis enteropathica and in normal adults assessed by whole-body counting technique. $\mathrm{Br}$ J Dermatol 1979;101(5):573-9.

2. Verhagen AR. Dermatoses in dark-skinned people. vol. 3. Basel: Ciba-Geigy 1976:p. 29.

3. Lonnerdal B, Stainslowski AG, Hurley LS. Isolation of a low molecular weight zinc binding ligand from human milk. J Inorg Biochem 1980;12(1):71-8.

4. Brandt T. Dermatitis in children with disturbances of the general condition and absorption of food. Acta Derm Venereol (Stockh) 1936;17:513-46.

5. Danbolt N, Closs K. Acrodermatitis enteropathica. Acta Derm Venereol (Stockh) 1942;23:127-69.

6. Ortega SS, Cachaza JA, Tovar IV, et al. Zinc deficiency dermatitis in parenteral nutrition: an electron-microscopic study. Dermatologica 1985;171(3):163-9.

7. Roohani $\mathrm{N}$, Hurrell $\mathrm{R}$, Kelishadi $\mathrm{R}$, et al. Zinc and its importance for human health: an integrative review. J Res Med Sci 2013;18(2):144-57.

8. Van Wouwe JP. Clinical and laboratory diagnosis of acrodermatitis enteropathica. Eur J Paediatr 1989;149(1):2-8.

9. Prasad AS. Laboratory diagnosis of zinc deficiency. J Am Coll Nutr 1985;4(6):591-8.

10. Kockaya G, Ufuktepe B, Kockaya PD, et al. The efficacy and undesired effects of high dosage zinc treatment. Journal of Applied Pharmaceutical Science 2012;2(3):157-9. 